\title{
Direct solution of uncertain bratu initial value problem
}

\author{
N. R. Anakira1, A. H. Shather ${ }^{2}$, A. F. Jameel ${ }^{3}$, A. K. Alomari ${ }^{4}$, A. Saaban ${ }^{5}$ \\ ${ }^{1}$ Department of Mathematics, Faculty of Science and Technology, Irbid National University, Jordan \\ ${ }^{2}$ Department of Comunication Engineering, Technical College of Enginnering, Sulaimani Polytechnic University, Iraq \\ ${ }^{4}$ Department of Mathematics, Faculty of Science, Yarmouk University, Jordan \\ ${ }^{3,5}$ School of Quantitative Sciences, Universiti Utara Malaysia (UUM), Malaysia
}

\begin{tabular}{|c|c|}
\hline Article Info & ABSTRACT \\
\hline Article history: & In this paper, an approximate analytical solution for solving the fuzzy Bratu \\
\hline Received Nov 3, 2018 & $\begin{array}{l}\text { equation based on variation iteration method (VIM) is analyzed and modified } \\
\text { without needed of any discretization by taking the benefits of fuzzy set }\end{array}$ \\
\hline Revised May 27, 2019 & theory. VIM is applied directly, without being reduced to a first order system, \\
\hline Accepted Jun 19, 2019 & $\begin{array}{l}\text { to obtain an approximate solution of the uncertain Bratu equation. } \\
\text { An example in this regard have been solved to show the capacity and }\end{array}$ \\
\hline
\end{tabular}

Analytical solution

Bratu equation

Fuzzy

Variation iteration method (VIM)

Copyright $@ 2019$ Institute of Advanced Engineering and Science. All rights reserved.

\section{Corresponding Author:}

N. R. Anakira,

Department of Mathematics,

Faculty of Science and Technology,

Irbid National University

2600 Irbid, Jordan.

Email: alanaghreh_nedal@yahoo.com

\section{INTRODUCTION}

In various fields of science and engineering, nonlinear phenomena are fundamental. However, the nonlinear models of real - life issues are still difficult to resolve numerically or analytically [1]. The research and development of better and more efficient solutions for approximate or numerical solution to non - linear problems has recently received considerable attention. Many of these nonlinear models are in the form of ordinary equations an often be understood by reference to a simple nonlinear ordinary initial value problem such as the Bratu equation. The Bratu type equation was derived from the thermal combustion theory models of solid fuel ignition [1] that has a number of physical applications, such as theory of chemical reactors, heat transmission, the electro station, the fluid mechanics, and the physical sciences [2]. Various known models classified as problems with dynamic real life that can be formulated as a mathematical model with fuzzy differential equations.

Fuzzy differential equations (FDEs) are a great instrument for modeling a dynamic system when insufficient information regarding its behaviour. The original FIVP problem occurred when these problems were imperfectly modeled and their character was under uncertainty. Fuzzy ordinary differential equations are a good way of modeling dynamic systems with uncertainties or vagueness. The fuzzy models are employed in several applications of real life, including population models [3, 4], mathematical physics [5], and medicine [6]. In many cases the initial value problem under fuzziness, its exact solution is not exist or very hard to obtain analytically. Therefore, developing the approximate methods from crisp domain to fuzzy domain is necessary in order to obtain the solution of nonlinear fuzzy problems. 
The approximate solutions to nonlinear fuzzy problems using approximate analytical methods usually helps to increase the perceptual perception of physical problems. The fundamental advantage of an approximate method is to solve strong nonlinear problems without the needs of the exact solution to determine the solution's accuracy. Several approximate analytical methods have been used in recent years to solve fuzzy initial problems. The Adomain Decomposition Method (ADM) is used for solving first order linear and nonlinear FIVP [7, 8] and second order linear FIVP [9]. Meanwhile, the Homotopy Perturbation Method (HPM) is used to solve first order linear and nonlinear FIVP [10, 11] and second order linear FIVP [12]. Furthermore, the Variational Iteration Method (VIM) [13] and Optimum Homotopy Asymptotic method [14] are used to solve first order nonlinear FIVP.VIM was first introduced and proposed by He [15-19]. This method is different from the some classical techniques whereby nonlinear equations are solved easily and more accurately. VIM has recently been applied to many fields in physics and engineering problems [20-22]. This method is very useful for solving high order linear and nonlinear initial value problem directly without reducing to a first system and requirement or restrictive assumptions for the nonlinear terms [17] compared to the HPM and ADM, where computational algorithms are normally used to decompose the nonlinear terms. In this study, we introduce the Bratu equation in fuzzy form for the first time and proposed the approximate analytical solution for second order nonlinear FIVP using VIM. Modified VIM will be the first time used for solving nonlinear Bratu equation and is analyzed based on the framework of fuzzy problem by taking the advantages of the fuzzy set theory.

\section{BASIC FUZZY CONCEPTS}

Definition 2.1 [23]: The $r$-level (or $r$-cut) set of a fuzzy set $\widetilde{\boldsymbol{U}}$, labeled as $\widetilde{\boldsymbol{U}}_{\boldsymbol{r}}$, is the crisp set of all $\boldsymbol{t} \in \boldsymbol{T}$ such that $\boldsymbol{\mu}_{\widetilde{U}} \geq \boldsymbol{r}$ i.e.

$$
\widetilde{U}_{r}=\left\{t \in T \mid \mu_{\widetilde{U}}>r, r \in[0,1]\right\} .
$$

The $r$-level set is the link between the fuzzy domain and the crisp domain by using the advantages of the theories in crisp domain in the fuzzy domain.

Figure 1 Shows the degree of fuzzy membership function [24] connected with the values of $r$-level sets for all $\boldsymbol{r} \in[\mathbf{0 , 1}]$. When the value of $r$-level set is approach to 1 , means that the fuzzy area become smaller and close to crisp domain.

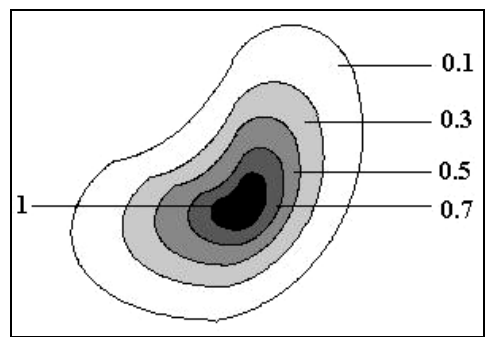

Figure 1. The fuzzy $r$-level sets

Definition 2.1: Fuzzy numbers are a subset of the real numbers set, and represent uncertain values. Fuzzy numbers are linked to degrees of membership that state how true it is to say if something belongs or not to a determined set. A fuzzy number $\mu$ is called a triangular fuzzy number [24] as shown in Figure 2 if defined by three numbers $\alpha<\beta<\gamma$ where the graph of $\mu(x)$ is a triangle with the base on the interval $[\alpha, \beta]$ and vertex at $x=\beta$ and its membership function has the following form,

$$
\mu(x ; \alpha, \beta, \gamma)=\left\{\begin{array}{rc}
0, & \text { if } x<\alpha \\
\frac{x-\alpha}{\beta-\alpha}, & \text { if } \alpha \leq x \leq \beta \\
\frac{\gamma-x}{\gamma-\beta}, & \text { if } \beta \leq x \leq y \\
0, & \text { if } x>\gamma
\end{array}\right.
$$




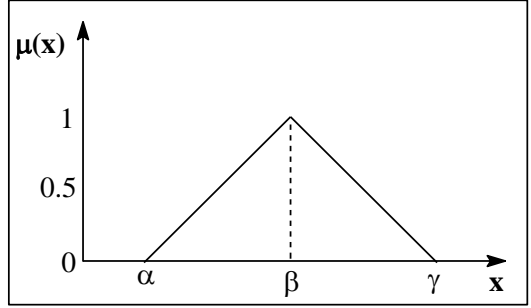

Figure 2. Triangular fuzzy number

With its $r$-level is: $[\mu]_{r}=[\alpha+r(\beta-\alpha), \gamma-r(\gamma-\beta)], r \in[0,1]$.

In this study the class of all fuzzy subsets of $\mathbb{R}$ will be denoted by $\tilde{E}$ and solution of FIVP satisfy the following properties [25]:

- $\mu(x)$ is normal, i.e $\exists x_{0} \in \mathbb{R}$ with $\mu\left(x_{0}\right)=1$,

- $\mu(x)$ Is convex fuzzy set, i. e. $\mu(\lambda x+(1-\lambda) t) \geq \min \{\mu(x), \mu(t)\} \forall x, t \in \mathbb{R}, \lambda[0,1]$,

- $\quad \mu$ upper semi-continuous on $\mathbb{R}$,

- $\quad \overline{\{t \in \mathbb{R}: \mu(x)>0\}}$ is compact.

$\tilde{E}$ is called the space of fuzzy numbers and $\mathbb{R}$ is a proper subset of $\tilde{E}$.

Define the $r$-level set $x \in \mathbb{R},[\mu]_{r}=\{x \backslash \mu(x) \geq r\}, 0 \leq r \leq 1$, where $[\mu]_{0}=\{x \backslash \mu(x)>0\}$ is compact, which is a closed bounded interval and denoted by $[\mu]_{r}=[\underline{\mu}(x), \bar{\mu}(x)]$. In the single parametric form [26], a fuzzy number is represented by an ordered pair of functions $[\underline{\mu}(x), \bar{\mu}(x)], r \in[0,1]$ which satisfies:

- $\underline{\mu}(x)$ is a bounded left continuous non-decreasing function over $[0,1]$.

- $\overline{\bar{\mu}}(x)$ is a bounded right continuous non-increasing function over $[0,1]$.

- $\underline{\mu}(x) \leq \bar{\mu}(x), r \in[0,1]$. A crisp number $r$ is simply represented by $\underline{\mu}(r)=\bar{\mu}(r)=r, r \in[0,1]$.

Definition 2.2 [25]: If $\tilde{E}$ be the set of all fuzzy numbers, we say that $f(x)$ is a fuzzy function if $f: \mathbb{R} \rightarrow \tilde{E}$

Definition 2.3 [27]: A mapping $f: T \rightarrow \tilde{E}$ for some interval $T \subseteq \tilde{E}$ is called a fuzzy function process and we denote $r$-level set by:

$$
[\tilde{f}(x)]_{r}=[\underline{f}(x ; r), \bar{f}(x ; r)], x \in K, r \in[0,1]
$$

The $r$-level sets of a fuzzy number are much more effective as representation forms of fuzzy set than the above. Fuzzy sets can be defined by the families of their $r$-level sets based on the resolution identity theorem.This is called the Zadeh extension principle.

Definition 2.4 [25]: Consider $\tilde{x}, \tilde{y} \in \tilde{E}$. If there exists $\tilde{z} \in \tilde{E}$ such that $\tilde{x}=\tilde{y}+\tilde{z}$, then $\tilde{z}$ is called the H-difference (Hukuhara difference) of $x$ and $y$ and is denoted by $\tilde{z}=\tilde{x} \ominus \tilde{y}$.

Definition 2.5 [26]: If $\tilde{f}: I \rightarrow \tilde{E}$ and $y_{0} \in I$, where $I \in\left[x_{0}, K\right]$. We say that $\tilde{f}$ Hukuhara differentiable at $y_{0}$, if there exists an element $\left[\widetilde{f}^{\prime}\right]_{r} \in \tilde{E}$ such that for all $h>0$ sufficiently small (near to 0 ), exists $\tilde{f}\left(y_{0}+h ; r\right) \ominus \tilde{f}\left(y_{0} ; r\right), \tilde{f}\left(y_{0} ; r\right) \ominus \tilde{f}\left(y_{0}-h ; r\right)$ and the limits are taken in the metric $(\tilde{E}, D)$ :

$$
\lim _{h \rightarrow 0+} \frac{\tilde{f}\left(y_{0}+h ; r\right) \Theta \tilde{f}\left(y_{0} ; r\right)}{h}=\lim _{h \rightarrow 0+} \frac{\tilde{f}\left(y_{0} ; r\right) \Theta \tilde{f}\left(y_{0}-h ; r\right)}{h}
$$

The fuzzy set $\left[\widetilde{f}^{\prime}\left(y_{0}\right)\right]_{r}$ is called the Hukuhara derivative of $\left[\widetilde{f}^{\prime}\right]_{r}$ at $y_{0}$.

These limits are taken in the space $(\widetilde{E}, D)$ if $x_{0}$ or $K$, then we consider the corresponding one-side derivation. Recall that $\tilde{x} \ominus \tilde{y}=\tilde{z} \in \tilde{E}$ are defined on $r$-level set, where $[\tilde{x}]_{r} \ominus[\tilde{y}]_{r}=[\tilde{z}]_{r}, \forall r \in[0,1]$. By consideration of definition of the metric $D$ all the $r$-level set $[\tilde{f}(0)]_{r}$ are Hukuhara differentiable at $y_{0}$, with Hukuhara derivatives $\left[\widetilde{f}^{\prime}\left(y_{0}\right)\right]_{r}$, when $\tilde{f}: I \rightarrow \tilde{E}$ is Hukuhara differentiable at $y_{0}$ with Hukuhara derivative $\left[\widetilde{f}^{\prime}\left(y_{0}\right)\right]_{r}$ it' lead to that $\tilde{f}$ is Hukuhara differentiable for all $r \in[0,1]$ which satisfies the above limits i.e. if $f$ is differentiable at $x_{0} \in\left[x_{0}+\alpha, K\right]$ then all its $r$-levels $\left[\widetilde{f^{\prime}}(x)\right]_{r}$ are Hukuhara differentiable at $x_{0}$. 
Definition 2.8 [28]: Define the mapping $\widetilde{f^{\prime}}: I \rightarrow \tilde{E}$ and $y_{0} \in \mathrm{I}$, where $I \in\left[x_{0}, K\right]$. We say that $\widetilde{f^{\prime}}$ Hukuhara differentiable $x \in \tilde{E}$, if there exists an element $\left[\tilde{f}^{(n)}\right]_{r} \in \tilde{E}$ such that for all $h>0$ sufficiently small (near to 0 ), exists $\tilde{f}^{(n-1)}\left(y_{0}+h ; r\right) \ominus \tilde{f}^{(n-1)}\left(y_{0} ; r\right), \tilde{f}^{(n-1)}\left(y_{0} ; r\right) \ominus \tilde{f}^{(n-1)}\left(y_{0}-h ; r\right)$ and the limits are taken in the metric $(\widetilde{E}, D)$

$$
\lim _{h \rightarrow 0+} \frac{\tilde{f}^{(n-1)}\left(y_{0}+h ; r\right) \ominus \tilde{f}^{(n-1)}\left(y_{0} ; r\right)}{h}=\lim _{h \rightarrow 0+} \frac{\tilde{f}^{(n-1)}\left(y_{0} ; r\right) \ominus \tilde{f}^{(n-1)}\left(y_{0}-h ; r\right)}{h}
$$

Exists and equal to $\tilde{f}^{(n)}$ and for $n=2$ we have second order Hukuhara derivative.

Theorem 2.2 [26]: Let $\tilde{f}:\left[x_{0}+\alpha, K\right] \rightarrow \widetilde{E}$ be Hukuhara differentiable and denote $\left[\widetilde{f}^{\prime}(x)\right]_{r}=$ $\left[\underline{f}^{\prime}(x), \bar{f}^{\prime}(x)\right]_{r}=\left[\underline{f}^{\prime}(x ; r), \bar{f}^{\prime}(x ; r)\right]$.Then the boundary functions $\underline{f}^{\prime}(x ; r), \bar{f}^{\prime}(x ; r)$ are differentiable we can write for second order fuzzy derivative

$$
\left[\tilde{\boldsymbol{f}}^{\prime \prime}(\boldsymbol{x})\right]_{\boldsymbol{r}}=\left[\left(\underline{\boldsymbol{f}}^{\prime \prime}(\boldsymbol{x} ; \boldsymbol{r})\right)^{\prime},\left(\overline{\boldsymbol{f}}^{\prime \prime}(\boldsymbol{x} ; \boldsymbol{r})\right)^{\prime}\right], \forall \boldsymbol{r} \in[\mathbf{0}, \mathbf{1}]
$$

Definition 2.5 [29, 30]: Each function $f: X \rightarrow Y$ induces another function $\tilde{f}: F(X) \rightarrow F(Y)$ defined for each fuzzy interval $U$ in $X$ by:

$$
\tilde{f}(U)(y)=\left\{\begin{array}{c}
\operatorname{Sup}_{x \in f^{-1}(y)} U(x), \text { if } y \in \operatorname{range}(f) \\
0 \quad, \text { if } y \notin \operatorname{range}(f)
\end{array}\right.
$$

This is also known as the Zadeh extension principle.

\section{GENERAL FUZZY VIM}

The formulation of VIM for of the approximate solution of crisp general differential equation was given in [15]. In this section, the new formulation of VIM for the general fuzzy differential equation by using the properties of the fuzzy definitions in section 2 will be presented. Consider general fuzzy differential equation

$$
L \tilde{y}(x)-N(\tilde{y}(x))=\tilde{h}(x)
$$

Where $L$ is a linear operator, $N$ a nonlinear operator of the fuzzy function $\tilde{y}(x)$ of crisp variable $x$ and $\tilde{h}(x)$ is the inhomogeneous term such that

$$
\begin{aligned}
& \tilde{y}(x)=\tilde{y}(x ; r)=[\underline{y}(x ; r), \bar{y}(x ; r)], \\
& \tilde{h}(x)=\tilde{h}(x ; r)=[\underline{h}(x ; r), \bar{h}(x ; r)], \text { for all level set } r \in[0,1] .
\end{aligned}
$$

According to VIM [15], a correction fuzzy function is constructed as follows

$$
\left\{\begin{array}{l}
\underline{y}_{i+1}(x ; r)=\underline{y}_{i}(x ; r)+\int_{t=0}^{x} \lambda\left\{L \underline{y}_{i}(t ; r)-N(\overbrace{y_{i}(t ; r)})-\underline{h}(t ; r)\right\} d t \\
\bar{y}_{i+1}(x ; r)=\bar{y}_{i}(x ; r)+\int_{t=0}^{x} \lambda\left\{L \bar{y}_{i}(t ; r)-N\left(\widetilde{\bar{y}_{i}(t ; r)}\right)-\bar{h}(t ; r)\right\} d t
\end{array}\right.
$$

for $=0,1,2, \ldots$. Here in (2) $\lambda$ is a Lagrange multiplier that can be identified optimally via variational iteration method [18]. According to [15] $\overbrace{\tilde{y}_{i}(t ; r)}$ is considered as restricted variation such that $\delta \overbrace{\tilde{y}_{i}(t ; r)}=0$. Expand (2) similar to VIM in crisp to get the following equations:

Lower bound solution

$$
\begin{aligned}
& \underline{y_{1}}(x ; r)=\underline{y}_{0}(x ; r)+\int_{t=0}^{x} \lambda\left\{L \underline{y}_{0}(t ; r)-N\left(\underline{y}_{0}(t ; r)\right)-\underline{h}(t ; r)\right\} d t \\
& \underline{y_{2}}(x ; r)=\underline{y}_{1}(x ; r)+\int_{t=0}^{x} \lambda\left\{L \underline{y}_{1}(t ; r)-N\left(\underline{y_{1}}(t ; r)\right)-\underline{h}(t ; r)\right\} d t \\
& \underline{y}_{3}(x ; r)=\underline{y}_{2}(x ; r)+\int_{t=0}^{x} \lambda\left\{L \underline{y}_{2}(t ; r)-N\left(\underline{y}_{2}(t ; r)\right)-\underline{h}(t ; r)\right\} d t
\end{aligned}
$$


Upper bound solution

$$
\begin{aligned}
& \bar{y}_{1}(x ; r)=\bar{y}_{0}(x ; r)+\int_{t=0}^{x} \lambda\left\{L \bar{y}_{0}(t ; r)-N\left(\bar{y}_{0}(t ; r)\right)-\bar{h}(t ; r)\right\} d t \\
& \bar{y}_{2}(x ; r)=\bar{y}_{1}(x ; r)+\int_{t=0}^{x} \lambda\left\{L \bar{y}_{1}(t ; r)-N\left(\bar{y}_{1}(t ; r)\right)-\bar{h}(t ; r)\right\} d t \\
& \bar{y}_{3}(x ; r)=\underline{y}_{2}(x ; r)+\int_{t=0}^{x} \lambda\left\{L \bar{y}_{2}(t ; r)-N\left(\bar{y}_{2}(t ; r)\right)-\bar{h}(t ; r)\right\} d t
\end{aligned}
$$

Where $\tilde{y}_{0}(x ; r)$ is an initial guess function with possible unknowns that satisfies the initial conditions of the given FIVP and $\lambda$ is the Lagrange multiplier optimally. The successive approximation, for $i \geq 0$ of the solution $\tilde{y}(x ; r)$ will be readily obtained upon using the determined the suitable Lagrange multiplier and selective of the initial approximation $\tilde{y}_{0}(x ; r)$ consequently, then the exact fuzzy solution is given by

$$
\tilde{Y}(x ; r)=\lim _{i \rightarrow \infty} \underline{y_{i}}(x ; r)
$$

\section{ANALYSIS OF THE FUZZY BRATU EQUATION}

According to [1] the general FIVP Bratu equation is given by:

$$
\left\{\begin{array}{c}
\widetilde{y}^{\prime \prime}(x)=\widetilde{a} e^{\widetilde{y}(x)}, x>0, \widetilde{a}>\widetilde{0} \\
\widetilde{y}(\mathbf{0})=\widetilde{b}, \widetilde{y}^{\prime}(0)=\tilde{c}
\end{array}\right.
$$

Following Section 2 to defuzzify (4) for all $\boldsymbol{r} \in[\mathbf{0 , 1}]$ as follows:

$\tilde{y}(x)$ : is a fuzzy function of the crisp variable $x$ such that $[\tilde{y}(x)]_{r}=[\underline{y}(x ; r), \bar{y}(x ; r)]$,

$[\tilde{a}]_{r}=[\underline{a}, \bar{a}]_{r},[\tilde{b}]_{r}=[\underline{b}, \bar{b}]_{r},[\tilde{c}]_{r}=[\underline{c}, \bar{c}]_{r}$ are triangular fuzzy numbers,

$\tilde{y}^{\prime \prime}(x):$ is the second order fuzzy H-derivative of such that $\left[\tilde{y}^{\prime \prime}(x)\right]_{r}=\left[\underline{y}^{\prime \prime}(x ; r), \bar{y}^{\prime \prime}(x ; r)\right]$,

Let $\tilde{f}(x, \tilde{y}(x ; r))=\tilde{a} e^{\tilde{y}(x)}$ such that $[\tilde{f}(x, \tilde{y}(x ; r))]_{r}=[\underline{f}(x, \tilde{y}(x ; r)), \bar{f}(x, \tilde{y}(x ; r))]$ then the following

$$
\left\{\begin{array}{l}
f(x, \tilde{y}(x ; r))=F_{1}[x, \underline{y}, \bar{y}]_{r} \\
\bar{f}(x, \tilde{y}(x ; r))=F_{2}[x, \underline{y}, \bar{y}]_{r}
\end{array}\right.
$$

Since $\tilde{y}^{\prime \prime}(x)=\tilde{f}(x, \tilde{y}(x ; r))$ by using the Zadeh extension principle in Section 2, we can define the following membership function

$$
\left\{\begin{array}{l}
f(x, \tilde{y}(x ; r))=\min \{\tilde{\mathcal{A}}(t, \tilde{\mu}(r)) \mid \tilde{\mu}(r) \in \tilde{y}(t ; r)\} \\
\bar{f}(x, \tilde{y}(x ; r))=\max \{\tilde{\mathcal{A}}(t, \tilde{\mu}(r)) \mid \tilde{\mu}(r) \in \tilde{y}(t ; r)\}
\end{array}\right.
$$

Where

$$
\left\{\begin{array}{l}
\underline{f}(x, \tilde{y}(x ; r))=F_{1}(x, \underline{y}(x ; r), \bar{y}(x ; r))=F_{1}(x, \tilde{y}(x ; r))=\underline{a}(r) e^{\underline{y}(x ; r)} \\
\bar{f}(x, \tilde{y}(x ; r))=F_{2}(x, \underline{y}(x ; r), \bar{y}(x ; r))=F_{2}(x, \tilde{y}(x ; r))=\bar{a}(r) e^{\bar{y}(x ; r)}
\end{array}\right.
$$

Finally (4) can be written as

$$
\begin{aligned}
& \left\{\begin{array}{c}
\underline{y}^{\prime \prime}(x ; r)=\underline{a}(r) e^{\underline{y}(x ; r)}, x>0, \tilde{a}>0 \tilde{0} \\
\underline{y}(0 ; r)=\underline{b}(r), \underline{y}^{\prime}(0 ; r)=\underline{c}(r)
\end{array}\right. \\
& \left\{\begin{array}{c}
\overline{\boldsymbol{y}}^{\prime \prime}(\boldsymbol{x} ; \boldsymbol{r})=\overline{\boldsymbol{a}}(\boldsymbol{r}) \boldsymbol{e}^{\overline{\boldsymbol{y}}(\boldsymbol{x} ; \boldsymbol{r}), \boldsymbol{x}>\mathbf{0}, \widetilde{\boldsymbol{a}}>\widetilde{\mathbf{0}}} \\
\overline{\boldsymbol{y}}(\mathbf{0} ; \boldsymbol{r})=\overline{\boldsymbol{b}}(\boldsymbol{r}), \overline{\boldsymbol{y}}^{\prime}(\mathbf{0} ; \boldsymbol{r})=\overline{\boldsymbol{c}}(\boldsymbol{r})
\end{array}\right.
\end{aligned}
$$


In this section, we present the solution of (8-9) by means of VIM in Section 3 and [1]. We first construct a correction functional

$$
\underline{y}_{i+1}(x ; r)=\underline{y}_{i}(x ; r)+\int_{t=0}^{x} \lambda(t)\left\{\underline{y}_{i}^{\prime \prime}(t ; r)-\underline{a}(r) e^{y(t ; r)}\right\} d t
$$

where $\overbrace{\underline{\boldsymbol{y}}(\boldsymbol{t} ; \boldsymbol{r})}$ are considered as restricted variations, which means $\overbrace{\underline{\boldsymbol{y}(\boldsymbol{t} ; \boldsymbol{r})}}=\mathbf{0}$. To obtain the suitable value of $\boldsymbol{\lambda}(\boldsymbol{t})$, we proceed as follows:

$$
\theta \underline{y}_{i+1}(x ; r)=\theta \underline{y}_{i}(x ; r)+\theta \int_{t=0}^{x} \lambda(t)\left\{\underline{y}_{i}^{\prime \prime}(t ; r)-\underline{a}(r) e^{y}(t ; r)\right\} d t
$$

and consequently

$$
\theta \underline{y}_{i+1}(x ; r)=\theta \underline{y}_{i}(x ; r)+\theta \int_{t=0}^{x} \lambda(t)\left\{\underline{y}_{i}^{\prime \prime}(t ; r)\right\} d t
$$

Integrating (12) by part which results the following

$$
\theta \underline{y}_{i+1}(x ; r)=\theta \underline{y}_{i}(x ; r)\left(1-\lambda^{\prime}(t)\right)+\theta \underline{y}_{i}(t ; r) \lambda(t)+\int_{t=0}^{x} \theta \underline{y}_{i}(t ; r) \lambda^{\prime \prime}(t) d t=0
$$

The stationary conditions can be obtained as follows:

$$
\left\{\begin{array}{c}
\left.\mathbf{1}-\lambda^{\prime}(\boldsymbol{t})=\mathbf{0}\right\}_{t=x} \\
\lambda(t)=\mathbf{0}\}_{t=x} \\
\left.\lambda^{\prime \prime}(t)=0\right\}_{t=x}
\end{array}\right.
$$

From (13), the Lagrange multipliers, therefore, can be identified as:

$$
\lambda(t)=t-x
$$

and the iteration VIM of the lower bound formula for solution of (4) is given as

$$
\underline{y}_{i+1}(x ; r)=\underline{y}_{i}(x ; r)+\int_{t=0}^{x}(t-x)\left\{\underline{y}_{i}^{\prime \prime}(t ; r)-\underline{a}(r) e^{y(t ; r)}\right\} d t
$$

Similarly for the upper bound solution of (4):

$$
\bar{y}_{i+1}(x ; r)=\bar{y}_{i}(x ; r)+\int_{t=0}^{x}(t-x)\left\{\bar{y}_{i}^{\prime \prime}(t ; r)-\bar{a}(r) e^{\bar{y}(x ; r)}\right\} d t
$$

\section{APPLICATION}

In this section, we employ the modified VIM in section to on FIVP Bratu equation and display the result in the form of tables and figures. According to Section 2, we consider the fuzzy version of the crisp initial value Bratu equation [2] for all $\boldsymbol{r} \in[\mathbf{0 , 1}]$ as follows

$$
\left\{\begin{array}{c}
\widetilde{y}^{\prime \prime}(x)=2 e^{\widetilde{y}(x)}, x>0 \\
\widetilde{y}(0)=[0.1 r-0.1,0.1-0.1 r], \widetilde{y}^{\prime}(0)=[0.1 r-0.1,0.1-0.1 r]
\end{array}\right.
$$

From the fuzzy analysis in section $4,(16)$ can be written as

$$
\begin{gathered}
\left\{\begin{array}{c}
\underline{y}^{\prime \prime}(x ; r)=2 e^{\underline{y}(x ; r)}, x>0 \\
\underline{y}(0 ; r)=0.1 r-0.1, \underline{y}^{\prime}(0 ; r)=0.1 r-0.1
\end{array}\right. \\
\left\{\begin{array}{c}
\overline{\boldsymbol{y}}^{\prime \prime}(\boldsymbol{x} ; \boldsymbol{r})=\mathbf{2} \boldsymbol{e}^{\overline{\boldsymbol{y}}(x ; \boldsymbol{r})}, \boldsymbol{x}>\mathbf{0} \\
\overline{\boldsymbol{y}}(\mathbf{0} ; \boldsymbol{r})=\mathbf{0 . 1}-\mathbf{0 . 1} \boldsymbol{1}, \overline{\boldsymbol{y}}^{\prime}(\mathbf{0} ; \boldsymbol{r})=\mathbf{0 . 1}-\mathbf{0 . 1} \boldsymbol{r}
\end{array}\right.
\end{gathered}
$$


According to Section 4 the VIM formula of the lower bound solution of (18) is:

$$
\underline{y}_{i+1}(x ; r)=\underline{y}_{i}(x ; r)+\int_{t=0}^{x}(t-x)\left\{\underline{y}_{i}^{\prime \prime}(t ; r)-2 e^{y(t ; r)}\right\} d t
$$

Where initial function of $(20)$ is ${\underline{y_{0}}}_{\mathbf{x}}(\boldsymbol{x})=\mathbf{0 . 1} \boldsymbol{r}-\mathbf{0 . 1}$. Similarly for the upper bound solution of (21) the VIM formula is

$$
\bar{y}_{i+1}(x ; r)=\bar{y}_{i}(x ; r)+\int_{t=0}^{x}(t-x)\left\{\bar{y}_{i}^{\prime \prime}(t ; r)-2 e^{\bar{y}(x ; r)}\right\} d t
$$

Where initial function of $(20)$ is $\overline{\boldsymbol{y}}_{\mathbf{0}}(\boldsymbol{x} ; \boldsymbol{r})=\mathbf{0 . 1}-\mathbf{0 . 1} \boldsymbol{r}$. Since VIM obtain the solution of initial value problems in the form of serious solution that converge to the exact solution [16], in order to obtain the convergence series we need to expand the nonlinear term $\boldsymbol{e}^{\widetilde{y}(\boldsymbol{x} ; \boldsymbol{r})}$ in (21) and (22) in to Maclaurin series of order three such that

$$
\left\{\begin{array}{l}
e^{\underline{y}(t ; r)}=1+\underline{y}(t ; r)+\frac{y(t ; r)^{2}}{2}+\frac{y(t ; r)^{3}}{6} \\
e^{\bar{y}(t ; r)}=1+\bar{y}(t ; r)+\frac{\bar{y}(t ; r)^{3}}{2}+\frac{\bar{y}(t ; r)^{3}}{6}
\end{array}\right.
$$

Substitute (23) in (21-22) to obtain the following VIM formula:

$$
\begin{aligned}
& \underline{y}_{i+1}(x ; r)=\underline{y}_{i}(x ; r)+\int_{t=0}^{x}(t-x)\left\{\underline{y}_{i}^{\prime \prime}(t ; r)-2\left(1+\underline{y}_{i}(t ; r)+\frac{y_{i}(t ; r)^{2}}{2}+\frac{y_{i}(t ; r)^{3}}{6}\right)\right\} d t \\
& \bar{y}_{i+1}(x ; r)=\bar{y}_{i}(x ; r)+\int_{t=0}^{x}(t-x)\left\{\bar{y}_{i}^{\prime \prime}(t ; r)-2\left(1+\bar{y}_{i}(t ; r)+\frac{\bar{y}_{i}(t ; r)^{3}}{2}+\frac{\bar{y}_{i}(t ; r)^{3}}{6}\right)\right\} d t
\end{aligned}
$$

Note that increasing the order of the series corresponding with the increasing of iteration of the approximate method the accuracy of the solution will be increase. As mentioned in Section 1 large number of nonlinear initial value problem under uncertainty cannot obtain the exact solution from its equation or very difficult to get the exact solution. Since the exact solution of (17) cannot be obtained analytically, in order to detect the accuracy of VIM approximate solution we define the following residual error [11], let VIM solution denoted by $\widetilde{\boldsymbol{y}}_{V I M}(\boldsymbol{x} ; \boldsymbol{r})$ such that

$$
\widetilde{R E}(x ; r)=\left|\widetilde{y}_{V I M}(x ; r)-2 e^{\widetilde{y}_{V I M}(x ; r)}\right|
$$

From (24) and (25) the third order VIM approximate solution of (17) for all $\boldsymbol{r} \in[\mathbf{0}, \mathbf{1}]$ is given by

$$
\widetilde{y}_{V I M}(x ; r)=\sum_{i=0}^{3} \tilde{y}_{i}(x ; r)
$$

Table 1 displayed the third order VIM approximate solution and the accuracy under the residual error (25) of (18) when $\boldsymbol{x}=\mathbf{0 . 1}$ for all fuzzy level sets $\boldsymbol{r} \in[\mathbf{0 , 1}$. In Table 1, also the VIM solution of (18) satisfies the properties of fuzzy set theory as mention in section 2 following the properties of single parametric form of fuzzy numbers.

Table 1. Third order VIM solution of (17) for all $\boldsymbol{r} \in[\mathbf{0 , 1}]$ at $\boldsymbol{x}=\mathbf{0 . 1}$

\begin{tabular}{ccccc}
\hline$r$ & $\underline{y}_{V I M}(0.1 ; r)$ & $\underline{R E}(0.1 ; r)$ & $\bar{y}_{V I M}(0.1 ; r)$ & $\overline{R E}(0.1 ; r)$ \\
\hline 0 & -0.09093798587602903 & 0.000005612044300384866 & 0.111072080117795350 & 0.000013004275519534758 \\
0.2 & -0.07075461470273547 & 0.000002075693933623057 & 0.090852466787663430 & 0.000005813502645946045 \\
0.4 & -0.05056754026676034 & $5.571770071988169 \times 10^{-7}$ & 0.070637204259720770 & 0.000002133090354128342 \\
0.6 & -0.03037668171833891 & $8.976426491003053 \times 10^{-8}$ & 0.050426211495021030 & $5.70827824564190 \times 10^{-7}$ \\
0.8 & -0.01018195818232265 & $2.174105673269366 \times 10^{-8}$ & 0.030219407483288146 & $9.43988887058822 \times 10^{-8}$ \\
1 & 0.0100167112423674850 & $2.343144123173601 \times 10^{-8}$ & 0.010016711242367485 & $2.34314412317360 \times 10^{-8}$ \\
\hline
\end{tabular}


The three dimensional Figure 3, shows that the third order VIM approximate solution for each value of $\boldsymbol{x} \in[\mathbf{0 , 0 . 1}]$ and fuzzy level sets $\boldsymbol{r} \in[\mathbf{0 , 1}]$ follows the properties of fuzzy numbers as mentioned in section 2 in the form of the triangular fuzzy number.

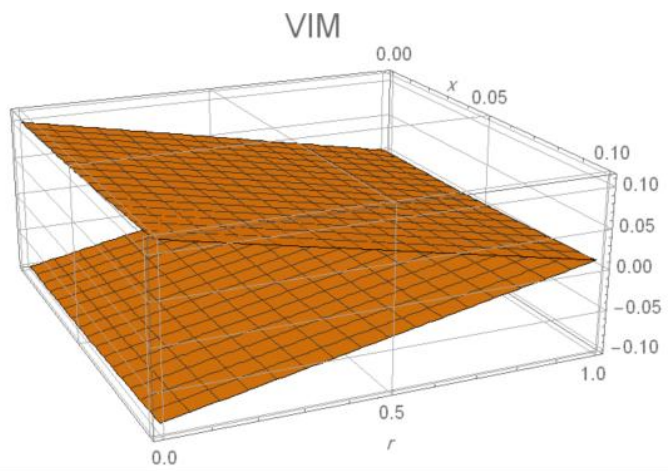

Figure 3. Third order VIM solution of (17) in the form of triangular fuzzy number for all $\boldsymbol{r} \in[\mathbf{0 , 1}]$ at $\boldsymbol{x} \in[\mathbf{0 , 0 . 1}]$

Since the exact solution cannot obtain in (18), Figure 4 shows sufficient accuracy with only third order VIM approximate lower and upper solution of (18) in terms of residual error (25) where the maximum error $\mathbf{1 0}^{-\mathbf{8}}$ for the lower solution and $\mathbf{1 0}^{-\mathbf{7}}$ for the upper solution for each value of $\boldsymbol{x} \in[\mathbf{0 , 0 . 1}]$ and fuzzy level sets $\boldsymbol{r} \in[\mathbf{0}, \mathbf{1}]$.
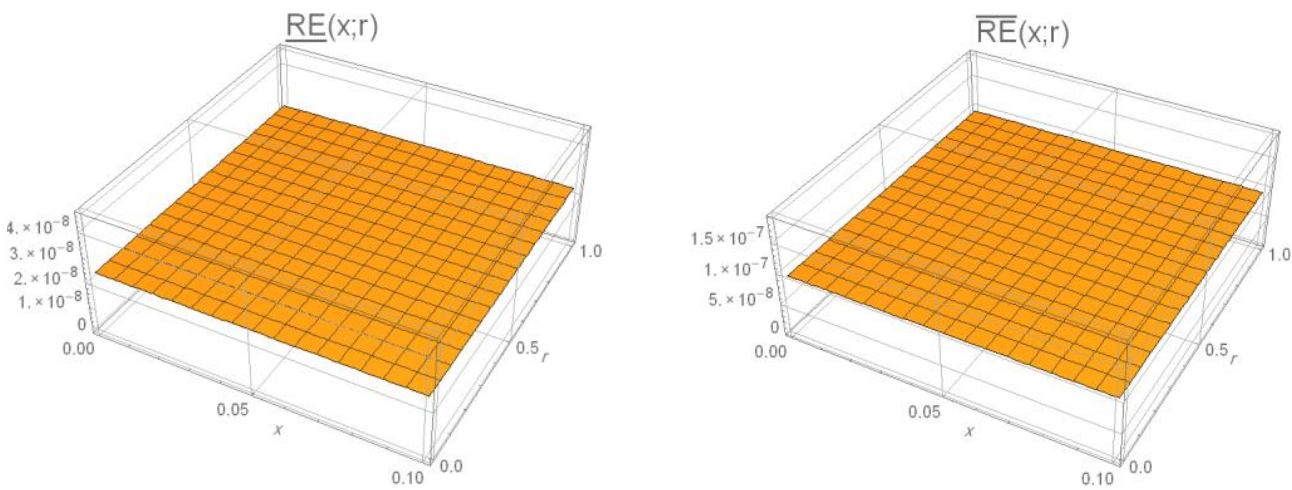

Figure 4. Accuracy of third order VIM solution of (17) for all $\boldsymbol{r} \in[\mathbf{0}, \mathbf{1}]$ at $\boldsymbol{x} \in[\mathbf{0 , 0 . 1}]$

\section{CONCLUSIONS}

In this research, an approximate analytical method was introduced to solve fuzzy differential equation in general form. A scheme based VIM to approximate the solution of the FIVP Bratu equation has been modified and implemented. An application involved Bratu equation with the fuzzy initial conditions show the efficiency of the proposed method. The research shows that implementation of VIM fast without decomposed the nonlinear terms of the given equation. The accuracy of VIM is determined without needed of an exact analytical solution. The Lagrangian multiplier of the FIVP Bratu equation has the same value for all $r$-level sets. All results of the experimental problem that obtained by VIM are satisfied the fuzzy number properties by taking the triangular shape.

\section{ACKNOWLEDGMENTS}

The authors are very grateful to the Ministry of Higher Education of Malaysia for providing us with the Fundamental Research Grant Scheme (FRGS) S/O number 14188 to enable us to pursue this research. 


\section{REFERENCES}

[1] B. Batiha, "Numerical Solution of Bratu-Type Equations by the Variational Iteration Method," Hacettepe Journal of Mathematics and Statistics, vol. 39(1), pp. 23-29, 2010.

[2] B. S. H. Kashkari and S. S. Z. Abbas, "Solution of Initial Value Prpblem of Bratu - Type Equation using Modifications of Homotopy Perturbation Method," International Journal of Computer Applications, vol. 162(5), pp. $0975-8887,2017$.

[3] A. Omer, O. Omer, "A Pray and Predator Model with Fuzzy Initial Values," Hacettepe Journal of Mathematics and Statistics, vol. 41(3), pp. 387-395, 2013.

[4] T. Smita, S. Chakraverty, "Numerical Solution of Fuzzy Arbitrary Order Predator-Prey Equations," Applications and Applied Mathematics, vol. 8(1), pp. 647-673, 2013.

[5] M. S. El Naschie, "From Experimental Quantum Optics to Quantum Gravity via a Fuzzy Kahler Manifold," Chaos. Soltion. Fract, vol. 25, pp. 969-977, 2005.

[6] M. F. Abbod, D. G. Von Keyserlingk, M. Mahfouf, "Survey Of Utilization Of Fuzzy Technology In Medicine And Healthcare," Fuzzy Set Systems, vol. 120, pp. 331-3491, 2001.

[7] E. Babolian, H. Sadeghi and S. Javadi, "Numerically Solution of Fuzzy Differential Equations by Adomian Method," Applied Mathematics and Computation, vol. 149, pp. 547-557, 2004.

[8] M. Ghanbari, "Solution of the first order linear fuzzy differential equations by some reliable methods," Journal of Fuzzy Set and Analysis, vol. 2012, pp. 1-20, 2012.

[9] L. Wang and S. Guo, "Adomian method for second-order fuzzy differential equation," International Journal of Mathematical and Computational Sciences, vol. 5(4), pp. 613-616, 2011.

[10] M. Ghanbari, "Numerical solution of fuzzy initial value problems under generalization differentiability by HPM," International Journal of Industrial Mathematics, vol. 1(1), pp. 19 - 39, 2009.

[11] A. F. Jameel, A. I. Md. Ismail, "Approximate Solution of First Order Nonlinear Fuzzy Initial Value Problem With Two Different Fuzzifications," Journal of Uncertain System, vol. 9(3), pp. 221-229, 2015.

[12] T. Smita, S. Chakraverty, "Numerical Solution of n-th Order Fuzzy Linear Differential Equations by Homotopy Perturbation Method," International Journal of Computer Applications, vol. 64(6), pp. 0975-8887, 2013.

[13] T. Allahviranloo, A. Panahi and H. Rouhparvar, "A computational method to find an approximate analytical solution for fuzzy differential equations," Analele Stiintifice Ale Universitatii Ovidius Constanta Seria Matematica, vol. 17(1), pp. 5-14, 2009.

[14] A. F. Jameel, A. Saaban, S. A. Altaie, N. R. Anakira, A. K. Alomari, N. Ahmad, "Solving First Order Nonlinear Fuzzy Differential Equations Using Optimal Homotopy Asymptotic Method," International Journal of Pure and Applied Mathematics, vol. 118(1), pp. 49-64, 2018.

[15] J. H. He, "Variational Iteration Method for Autonomous Ordinary Differential Systems," Applied Mathematics and Computation, vol. 114, pp. 115-123, 2000.

[16] J. H. He, "Variational Iteration Method- A Kind of Non-Linear Analytical Technique: Some Examples," International Journal of Non-Linear Mechanics, vol. 34, pp. 699-708, 1999.

[17] J. H. He, "Variational Iteration Method for Delay Differential Equations," Communications in Nonlinear Science and Numerical Simulation, vol. 2, pp. 235-236, 1997.

[18] J. H. He, "Variational Iteration Method for Autonomous Ordinary Differential Systems," Applied Mathematics and Computation, vol. 114, pp. 115-123, 2000.

[19] A. Bibi and F. Merahi, "Adomian Decomposition Method Applied To Linear Stochastic Differential Equations," International Journal of Pure and Applied Mathematics, vol. 118(3), pp. 501-510, 2018.

[20] H. Aminikhah and J. Biazar, "A New HPM For Ordinary Differential Equations," Numerical Methods for Partial Differential Equations, vol. 26(2), pp. 480-489, 2009.

[21] K. Hammood Al-jizani, N. Atinah Ahmad, F. S. Fadhel, "Variational Iteration Method for Solving Riccati Matrix Differential Equations," International Journal of Electrical, Electronics and Computer Systems (IJEECS), vol. 5(3), pp. 673- 683, 2017.

[22] A. M. Wazwaz, "The variational iteration method for solving two forms of Blasius equation on a half-infinite domain," Applied Mathematics and Computations, vol. 188, pp. 485-491, 2007.

[23] S. Bodjanova, "Median Alpha-Levels of A Fuzzy Number," Fuzzy Sets and Systems, vol. 157(7), pp. 879-91, 2006.

[24] D. Dubois, H. Prade, "Towards fuzzy differential calculus, Part 3: Differentiation," Fuzzy Sets and Systems, vol. 8, pp. 225-233, 1982.

[25] O. S. Fard, "An iterative scheme for the solution of generalized system of linear fuzzy differential equations," World Applied Sciences Journal, vol. 7, pp. 1597-11604, 2009.

[26] R. Faranak, I. Fudziah, A. Ali, S. Soheil, "Numerical solution of second-order fuzzy differential equation using improved Runge-Kutta Nystrom method," Mathematical Problems in Engineering, vol. 2013, pp. 1-10, 2013.

[27] O. Kaleva, "Fuzzy differential equation," Fuzzy Sets and Systems, vol. 24, pp. 301-317, 1987.

[28] S. S. Mansour, N. Ahmady, "A numerical method for solving Nth-order fuzzy differential equation by using Characterization theorem," Communications in Numerical Analysis, vol. 2012, pp. 1-12, 2012.

[29] L .A. Zadeh, "Fuzzy sets," Information and Control, vol. 8, pp. 338-353, 1965.

[30] L .A. Zadeh, "Toward a generalized theory of uncertainty," Information Sciences, vol. 172(2), pp. 1-40, 2005. 